\title{
Modeling accounting year dependence in runoff triangles
}

\section{Journal Article}

Author(s):

Salzmann, Robert; Wüthrich, Mario V.

Publication date:

2012-12

Permanent link:

https://doi.org/10.3929/ethz-b-000060149

Rights / license:

In Copyright - Non-Commercial Use Permitted

Originally published in:

European Actuarial Journal 2(2), https://doi.org/10.1007/s13385-012-0055-3 


\title{
Modeling accounting year dependence in runoff triangles
}

\author{
Robert Salzmann • Mario V. Wüthrich
}

Received: 9 December 2011/Revised: 25 May 2012/Accepted: 31 July 2012/

Published online: 6 September 2012

(C) DAV / DGVFM 2012

\begin{abstract}
Typically, non-life insurance claims data is studied in claims development triangles which display the two time axes accident years and development years. Most stochastic claims reserving models assume independence between different accident years. Therefore, such models fail to model claims inflation appropriately, because claims inflation acts on all accident years simultaneously. We introduce a Bayes chain ladder reserving model which enables us to model claims inflation. In this model we derive analytical formulas for the posterior distribution, the claims reserves and their prediction uncertainty.
\end{abstract}

Keywords Accounting year effects modeling - Claims inflation - Bayes chain ladder model · Multivariate dependence modeling - General insurance liabilities . Outstanding loss liabilities · Claims reserving · Loss development

\section{Introduction}

Non-life insurance claims reserving is often based on data generated by a flow of information arriving from the development of insurance claims. The observed data is recorded in so-called claims development triangles which display the two time axes accident years and development years. The claims entries on the same diagonals in these claims development triangles correspond to observations in the same

R. Salzmann $(\bowtie) \cdot$ M. V. Wüthrich

RiskLab, Department of Mathematics, ETH Zurich,

8092 Zurich, Switzerland

e-mail: robert.salzmann@math.ethz.ch

M. V. Wüthrich

e-mail: mario.wuethrich@math.ethz.ch 
accounting years. Most stochastic claims reserving models assume independence between different accident years (along the accident year axis). However, claims registered in the same accounting year may be subject to accounting year effects such as claims inflation (see e.g. Venter [16] and Clark [2]) or other external factors. Consequently, accounting year effects influence all accident years simultaneously which implies dependence between different accident years. Therefore, the common assumption that accident years behave independently is often violated in practice and models making that assumption are miss-specified when accounting year effects exist. This and the fact that claims inflation is a main risk driver in claims reserving give rise to investigate claims reserving models that allow for the modeling of accounting year effects. More precisely, we aim to model changing effects and trends along accounting years. On the contrary, as discussed in Kuang et al. [11], modeling persistent constant trends can be tackled by standard chain ladder (CL) type models.

In the existing actuarial literature accounting year effects modeling is studied only sparsely. A recent paper by Wüthrich [17] studies an extension of the gammagamma Bayes CL model that allows for Bayesian inference on accounting year effects. Moreover, we also refer to that paper for a summary on the existing actuarial literature on accounting year effects and claims inflation modeling. Within this context we further refer to de Jong [7], Jessen and Rietdorf [6] and a recent series of papers by Kuang et al. [9, 10] and [11] who study an extended CL-type model based on accident year, development year and accounting year parameters. The latter describes parameter estimation within a maximum likelihood framework and then discusses the identification problem when having a crossclassified model.

In this paper we define a multivariate Bayes CL model that allows for the modeling of dependence along accounting years. In contrast to the existing literature we are able to derive closed form solutions for the posterior distribution, the claims reserves and the corresponding prediction uncertainty. The closed form solutions enable us to study sensitivities of claims reserves and the corresponding prediction uncertainty as a function of a correlation parameter within accounting years. A case study shows that modeling accounting year dependence in runoff triangles reduces diversification effects between accident years which substantially contributes to the prediction uncertainty.

Organization of the paper. In Sect. 2 we define the multivariate Bayes CL model. In Sect. 3 we calculate unconditional and posterior distributions. The ultimate claim prediction and the corresponding conditional mean square error of prediction (MSEP) are calculated in Sect. 4. Section 5 presents a case study where the results are evaluated by the means of a real data example. Finally, we conclude in Sect. 6. Proofs are provided in the appendix.

\section{Model assumptions}

We denote cumulative claims by $C_{i, j}$, where $i \in\{1, \ldots, I\}$ corresponds to the accident year and $j \in\{0, \ldots, J\}$ to the development year. We assume that $I \geq J+1$ 
and that all claims are settled after development year $J$, then $C_{i, J}$ denotes the total ultimate claim of accident year $i$. For $k \geq 1$ the period $(k-1, k]$ denotes accounting year $k$. The cumulative claims $C_{i, j}$ with $i+j=k$ are all observed at the end of the same accounting year $k$. At time $I$, i.e. at the end of accounting year $I$, we have information (observations) in the upper left triangle/trapezoid

$$
\mathcal{D}_{I}=\left\{C_{i, j} ; i+j \leq I, i=1, \ldots, I, j=0, \ldots, J\right\},
$$

and we aim to predict the lower right triangle

$$
\mathcal{D}_{I}^{c}=\left\{C_{i, j} ; i+j>I, i=1, \ldots, I, j=0, \ldots, J\right\},
$$

i.e. the inexperienced part of the claims development at time $I$, see Fig. 1 which shows a so-called runoff triangle.

Accounting year effects influence the diagonals of the runoff triangle, see Fig. 1. Therefore, we stack all cumulative claims $C_{i, j}$ that belong to the same accounting year into one vector. For $k=1, \ldots, I+J$ we define the diagonal vectors $c^{(k)}$ by

$$
\boldsymbol{c}^{(k)}=\left(C_{k \wedge I,(k-I) \vee 0}, \ldots, C_{(k-J) \vee 1,(k-1) \wedge J}\right)^{\prime} \in \mathbb{R}^{q},
$$

where $q=q(k)=\min \{k,(J+1),(I+J+1-k)\} \in\{1, \ldots, J+1\}$ and $x \wedge y=$ $\min \{x, y\}$, and $x \vee y=\max \{x, y\}$. The elements of these diagonal vectors are all subject to the same diagonal effects. In Fig. 1 we highlight $c^{(I)}$, which is the last observed diagonal at time $I$. Note that the diagonal vectors $\boldsymbol{c}^{(k)}$ are of different length for different accounting years $k$. For increasing $k$ and $k \leq I$ their length increases, having maximal length for $k=I$ and afterwards decreases for $k>I$. We use (2.1) as a generic definition for diagonal vectors, see also (2.3) below.

Similar as in Hertig's model, see Hertig [5] and de Jong [7], we define individual incremental log-link ratios $\xi_{i, j}$ by

$$
\xi_{i, j}=\log \left(C_{i, j} / C_{i, j-1}-1\right)
$$

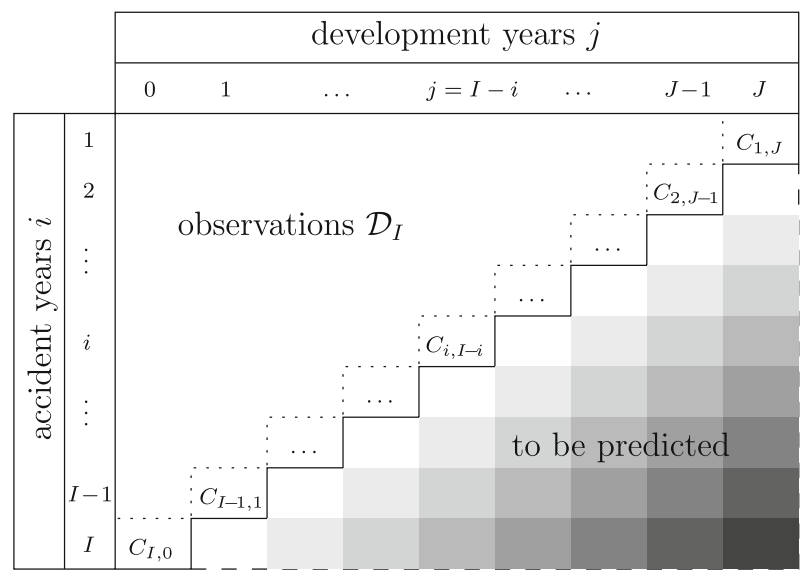

Fig. 1 Runoff triangle, the lower right triangle has to be predicted (taking possible dependencies along accounting years into account) 
for $j \geq 0$ with $C_{i,-1} \equiv 1$. Hence, subsequent cumulative claims in accident year $i$ are linked by the following relation

$$
C_{i, j}=C_{i, j-1}\left(\exp \left\{\xi_{i, j}\right\}+1\right) .
$$

In analogy to the cumulative claims $\boldsymbol{c}^{(k)}$ of accounting year $k$ we consider the vectors

$$
\boldsymbol{\xi}^{(k)}=\left(\xi_{k \wedge I,(k-I) \vee 0}, \ldots, \xi_{(k-J) \vee 1,(k-1) \wedge J}\right)^{\prime} \in \mathbb{R}^{q} .
$$

As a modification of Hertig's model we define the following multivariate Bayes CL model that allows one to model dependencies between the elements of the random vectors $\boldsymbol{\xi}^{(k)}$. A similar approach is presented in de Jong [7] where Hertig's model is extended to model accident year, development year or accounting year correlation on the basis of time series models.

Model 1 [Bayes $C L$ model with accounting year correlation] Let $\rho \in[0,1)$ and $\boldsymbol{\sigma}=\left(\sigma_{0}, \ldots, \sigma_{J}\right)^{\prime}$, with $\sigma_{j}>0$ for all $j \in\{0, \ldots, J\}$, be fixed parameters. Then

- conditionally, given $\boldsymbol{\mu}=\left(\mu_{0}, \ldots, \mu_{J}\right)^{\prime} \in \mathbb{R}^{J+1}$, we assume that $\boldsymbol{\xi}^{(1)}, \ldots, \boldsymbol{\xi}^{(I+J)}$ are independent and multivariate normally distributed with

$$
\left.\boldsymbol{\xi}^{(k)}\right|_{\boldsymbol{\mu}} \sim \mathcal{N}\left(\boldsymbol{\mu}^{(k)}, \Sigma_{k}\right)
$$

where the mean vector $\boldsymbol{\mu}^{(k)}$ is given by

$$
\boldsymbol{\mu}^{(k)}=\left(\mu_{(k-I) \vee 0}, \ldots, \mu_{(k-1) \wedge J}\right)^{\prime} \in \mathbb{R}^{q}
$$

and the covariance matrix $\Sigma_{k}$ is given by

$$
\Sigma_{k}=\left(\sigma_{m} \sigma_{n}\left(1_{\{m=n\}}+\rho 1_{\{m \neq n\}}\right)\right)_{m, n=(k-I) \vee 0, \ldots,(k-1) \wedge J} \in \mathbb{R}^{q \times q} .
$$

- $\boldsymbol{\mu}$ is multivariate normally distributed having prior mean vector $\boldsymbol{\phi}=\left(\phi_{0}, \ldots\right.$, $\left.\phi_{J}\right)^{\prime} \in \mathbb{R}^{J+1}$ and positive definite prior covariance matrix $T \in \mathbb{R}^{(J+1) \times(J+1)}$.

\section{Remark 1}

- Model 1 is a so-called Bayes CL model. It provides a mathematically consistent framework to incorporate prior expert knowledge and observed data into the model. Moreover, it accounts for the parameter uncertainty of $\boldsymbol{\mu}$ in a canonical way.

- The parameters $\sigma_{j}$ and $\rho$ are chosen to be fixed constants. Full Bayes models, also modeling $\sigma_{j}$ and $\rho$ stochastically, are no feasible alternative since we would like to have closed form solutions for unconditional and posterior densities, as provided in Sect. 3. However, more complex models can be evaluated using Markov chain Monte Carlo (MCMC) simulation methods, but then the study of sensitivities in $\rho$ becomes more involved. The same remark holds true if we replace the distributional assumptions in Model 1 by other distributions. 
- From Eq. (2.2) we see that the term $\left(\exp \left\{\xi_{i, j}\right\}+1\right)$ plays the role of the individual link factor in the CL context. That is, $\exp \left\{\xi_{i, j}\right\}$ corresponds to the incremental share of the individual link ratio. Therefore, Model 1 accounts for the fact that accounting year effects only affect future payments if $C_{i, j}$ denote cumulative payments, see also Wüthrich [17]. Moreover, this implies that for Model 1 to be fulfilled incremental claims have to be non-negative, i.e. $C_{i, j+1}-C_{i, j} \geq 0$.

- As a special property of the multivariate normal distribution we highlight that zero correlation implies independence. Therefore, Model 1 with $\rho=0$ provides the case where accident years behave conditionally independently, which corresponds to Hertig's log-normal model, (see Hertig [5]). We use the independent case as the benchmark model. In fact, in Model 1 we choose notation $\xi^{(k)}$ to highlight the nature of dependencies along diagonals. For $\rho=0$ one should rather use the classical display as used in Hertig [5].

- Our aim is to study sensitivities of claims reserves and the corresponding prediction uncertainty as a function of the accounting year correlation parameter $\rho \in[0,1)$.

- One could aim to replace the multivariate normal assumption in Model 1 by an elliptical model. However, this model will in general not allow for closed form solutions, see Section 3.3.3 in McNeil et al. [13]. Other interesting extensions are so-called hierarchical generalized linear models (which also need to be solved numerically), see Gigante et al. [4].

\section{Unconditional and posterior distributions}

To keep notation simple we first give the following preliminary definitions.

We define the projection $P_{k}$ that maps $\boldsymbol{\mu}$ onto $\boldsymbol{\mu}^{(k)}$. That is, we define $P_{k}: \mathbb{R}^{J+1} \rightarrow$ $\mathbb{R}^{q}$ by $\boldsymbol{\mu} \mapsto P_{k} \boldsymbol{\mu}=\boldsymbol{\mu}^{(k)}$. Since $P_{k}$ is a linear transformation we identify $P_{k}$ with the corresponding $(q \times(J+1))$-matrix. Model 1 then implies that

$$
\left.\boldsymbol{\xi}^{(k)}\right|_{\boldsymbol{\mu}} \sim \mathcal{N}\left(P_{k} \boldsymbol{\mu}, \Sigma_{k}\right)
$$

for all $k=1, \ldots, I+J$. Moreover, we define the concatenated vector $\boldsymbol{\xi}=\left(\boldsymbol{\xi}^{(1)^{\prime}}, \ldots, \boldsymbol{\xi}^{(I+J)^{\prime}}\right)^{\prime} \in \mathbb{R}^{I(J+1)}$. The conditional independence of the vectors $\boldsymbol{\xi}^{(k)}$, given $\boldsymbol{\mu}$, and the distributional assumptions of Model 1 allow one to derive the unconditional distribution of the vector $\boldsymbol{\xi}$. That is, instead of conditioning on $\boldsymbol{\mu}$, we state the unconditional distribution of $\xi$ directly as a function of the model parameters $\sigma, \phi, T$ and $\rho$.

Via the moment generating function of $\xi$ we determine the unconditional distribution of $\xi$ which is provided in the following theorem. 
Theorem 2 Under the assumptions of Model 1, the unconditional distribution of $\boldsymbol{\xi}$ is multivariate normal $\mathcal{N}(\varphi, \Upsilon)$ with mean and covariance matrix given by

$$
\begin{aligned}
& \boldsymbol{\varphi}=\left(\left(P_{1} \boldsymbol{\phi}\right)^{\prime}, \ldots,\left(P_{I+J} \phi\right)^{\prime}\right)^{\prime} \in \mathbb{R}^{I(J+1)}, \quad \text { and } \\
& \Upsilon=\left(\begin{array}{ccc}
\Upsilon_{1,1} & \cdots & \Upsilon_{1,(I+J)} \\
\vdots & & \vdots \\
\Upsilon_{(I+J), 1} & \cdots & \Upsilon_{(I+J),(I+J)}
\end{array}\right) \in \mathbb{R}^{I(J+1) \times I(J+1)},
\end{aligned}
$$

with $\Upsilon_{k, l}=P_{k} T P_{l}^{\prime}+\Sigma_{k} 1_{\{k=l\}} \in \mathbb{R}^{q(k) \times q(l)}$, i.e. $\Upsilon_{k, l}$ corresponds to the covariance matrix of the vectors $\xi^{(k)}$ and $\xi^{(l)}$ for $k, l=1, \ldots, I+J$.

Assume that we have observations $\mathcal{D}_{I}$, i.e. we have observed the incremental loglink ratios of the upper left trapezoid given by $\boldsymbol{\xi}_{\mathcal{D}_{I}}=\left(\boldsymbol{\xi}^{(1)^{\prime}}, \ldots, \boldsymbol{\xi}^{(I)^{\prime}}\right)^{\prime} \in \mathbb{R}^{d}$, with $d=\sum_{k=1}^{I} q(k)$. The unobserved incremental log-link ratios of the lower right triangle $\mathcal{D}_{I}^{c}$ are given by $\xi_{\mathcal{D}_{I}^{c}}=\left(\xi^{(I+1)^{\prime}}, \ldots, \xi^{(I+J)^{\prime}}\right)^{\prime} \in \mathbb{R}^{c}$, with $c=I(J+1)-d$. Consequently, we would like to derive the distribution of $\xi_{\mathcal{D}_{I}^{c}}$, given $\xi_{\mathcal{D}_{I}}$, i.e. we would like to do Bayesian inference on $\boldsymbol{\xi}_{\mathcal{D}_{I}^{c}}$, given $\boldsymbol{\xi}_{\mathcal{D}_{I}}$.

Given $\mathcal{D}_{I}$, the vectors $\xi_{\mathcal{D}_{I}}$ and $\xi_{\mathcal{D}_{I}^{c}}$ split the vector $\xi=\left(\xi_{\mathcal{D}_{I}}^{\prime}, \xi_{\mathcal{D}_{I}^{c}}^{\prime}\right)^{\prime}$ into an observed and an unobserved part. Accordingly, we split the covariance matrix $\Upsilon$ of Theorem 2 into

$$
\begin{aligned}
\Upsilon_{\mathcal{D}_{I}} & =\left(\Upsilon_{m, n}\right)_{m, n=1, \ldots, I} \in \mathbb{R}^{d \times d}, \\
\Upsilon_{\mathcal{D}_{I}^{c}} & =\left(\Upsilon_{m, n}\right)_{m, n=I+1, \ldots, I+J} \in \mathbb{R}^{c \times c} \quad \text { and } \\
\Upsilon_{\mathcal{D}_{I}^{c}, \mathcal{D}_{I}}^{\prime} & =\Upsilon_{\mathcal{D}_{I}, \mathcal{D}_{I}^{c}}=\left(\Upsilon_{m, n}\right)_{m=1, \ldots, I, n=I+1, \ldots, I+J} \in \mathbb{R}^{d \times c},
\end{aligned}
$$

which implies the representation

$$
\Upsilon=\left(\begin{array}{c|c}
\Upsilon_{\mathcal{D}_{I}} & \Upsilon_{\mathcal{D}_{I}, \mathcal{D}_{I}^{c}} \\
\hline \Upsilon_{\mathcal{D}_{I}^{c}, \mathcal{D}_{I}} & \Upsilon_{\mathcal{D}_{I}^{c}}
\end{array}\right) \in \mathbb{R}^{I(J+1) \times I(J+1)}
$$

Moreover, we define the corresponding means

$$
\begin{aligned}
& \boldsymbol{\varphi}_{\mathcal{D}_{I}}=\left(\left(P_{1} \boldsymbol{\phi}\right)^{\prime}, \ldots,\left(P_{I} \boldsymbol{\phi}\right)^{\prime}\right)^{\prime} \in \mathbb{R}^{d}, \quad \text { and } \\
& \boldsymbol{\varphi}_{\mathcal{D}_{I}^{c}}=\left(\left(P_{I+1} \boldsymbol{\phi}\right)^{\prime}, \ldots,\left(P_{I+J} \boldsymbol{\phi}\right)^{\prime}\right)^{\prime} \in \mathbb{R}^{c} .
\end{aligned}
$$

The following corollary is a direct consequence of standard multivariate normal analysis, see for instance Result 4.6 in Johnson and Wichern [8].

Corollary 1 Under the assumptions of Model 1 we have

$$
\left.\boldsymbol{\xi}_{\mathcal{D}_{I}^{c}}\right|_{\xi_{\mathcal{D}_{I}}} \sim \mathcal{N}\left(\varphi^{\text {post }}, \Upsilon^{\text {post }}\right)
$$


with the first two conditional moments given by

$$
\begin{aligned}
& \varphi^{\text {post }}=\boldsymbol{\varphi}_{\mathcal{D}_{I}^{c}}+\Upsilon_{\mathcal{D}_{I}^{c}, \mathcal{D}_{I}} \Upsilon_{\mathcal{D}_{I}}^{-1}\left(\xi_{\mathcal{D}_{I}}-\varphi_{\mathcal{D}_{I}}\right), \quad \text { and } \\
& \Upsilon^{\text {post }}=\Upsilon_{\mathcal{D}_{I}^{c}}-\Upsilon_{\mathcal{D}_{I}^{c}, \mathcal{D}_{I}} \Upsilon_{\mathcal{D}_{I}}^{-1} \Upsilon_{\mathcal{D}_{I}, \mathcal{D}_{I}^{c}} .
\end{aligned}
$$

\section{Remark.}

- The posterior covariance matrix $\Upsilon^{\text {post }}$ is the so-called Schur complement of $\Upsilon_{\mathcal{D}_{I}}$ in the covariance matrix $\Upsilon$.

- Corollary 1 allows one to calculate the conditional expectation of the ultimate claim and the corresponding conditional MSEP in closed form which is presented in Sect. 4. This is a major advantage compared to the model studied in Wüthrich [17].

- From Corollary 1 we can directly simulate the ultimate claim $C_{i, J}$, given $\mathcal{D}_{I}$, by simple Monte Carlo (MC) simulation techniques. From this predictive distribution we can calculate any other risk measure such as, for instance, the Value-at-Risk or the expected shortfall.

\section{Ultimate claim prediction and prediction uncertainty}

In this section we study the prediction of the ultimate claim and the uncertainty thereof. We start with some preliminary definitions and results. For single accident years $i$ we define the ultimate claim predictor by $\widehat{C}_{i, J}=\mathbb{E}\left[C_{i, J} \mid \mathcal{D}_{I}\right]$ and for aggregated accident years we define

$$
\widehat{C}_{J}=\mathbb{E}\left[\sum_{i=I-J+1}^{I} C_{i, J} \mid \mathcal{D}_{I}\right]=\sum_{i=I-J+1}^{I} \widehat{C}_{i, J}
$$

According to Eq. (2.2) we can write $C_{i, J}$ for $i>I-J$ as follows

$$
C_{i, J}=C_{i, I-i} \prod_{j=I-i+1}^{J}\left(\exp \left\{\xi_{i, j}\right\}+1\right),
$$

where $C_{i, I-i}$ is observed at time $I$.

In a next step we express formula (4.2) in terms of $\boldsymbol{\xi}_{\mathcal{D}_{I}^{c}}$. For $i>I-J$ we define the projection $A_{i}: \mathbb{R}^{c} \rightarrow \mathbb{R}^{J-(I-i)}$ by $\boldsymbol{\xi}_{\mathcal{D}_{I}^{c} \mapsto A_{i}} \boldsymbol{\xi}_{\mathcal{D}_{I}^{c}}=\left(\xi_{i, I-i+1}, \ldots, \xi_{i, J}\right)^{\prime}$. That is, $A_{i}$ projects $\xi_{\mathcal{D}_{I}^{c}}$ onto the vector with the components corresponding only to accident year $i$ in the lower right triangle $\mathcal{D}_{I}^{c}$. Since $A_{i}$ is a linear transformation we identify $A_{i}$ with a $((J-(I-i)) \times c)$-matrix.

Furthermore, we define $\tilde{\boldsymbol{e}}_{i, s}$ for $s \in\left\{1, \ldots, 2^{J-(I-i)}\right\}$ to be all different binary sequences of length $J-(I-i)$ written as vectors, i.e.

$$
\tilde{\boldsymbol{e}}_{i, s} \in\left\{\left(x_{1}, \ldots, x_{J-(I-i)}\right)^{\prime} \mid x_{v} \in\{0,1\}, \forall v=1, \ldots, J-(I-i)\right\} .
$$


By setting $\boldsymbol{e}_{i, s}=\left(\tilde{\boldsymbol{e}}_{i, s}^{\prime} A_{i}\right)^{\prime} \in \mathbb{R}^{c}$, formula (4.2) can be rewritten as follows

$$
C_{i, J}=C_{i, I-i} \prod_{j=I-i+1}^{J}\left(\exp \left\{\xi_{i, j}\right\}+1\right)=C_{i, I-i} \sum_{s=1}^{2^{J-(I-i)}} \exp \left\{\boldsymbol{e}_{i, s}^{\prime} \boldsymbol{\xi}_{\mathcal{D}_{I}^{c}}\right\} .
$$

Formula (4.3) together with Corollary 1 allow one to derive a closed form solution for the ultimate claim predictor, given $\mathcal{D}_{I}$, which is provided in the following theorem.

Theorem 3 For $i>I-J$ and under the assumptions of Model 1 we obtain for the ultimate claim predictor $\widehat{C}_{i, J}$

$$
\widehat{C}_{i, J}=C_{i, I-i} \sum_{s=1}^{2^{J-(I-i)}} \exp \left\{\boldsymbol{e}_{i, s}^{\prime} \boldsymbol{\varphi}^{\text {post }}+\frac{1}{2} \boldsymbol{e}_{i, s}^{\prime} \Upsilon^{p o s t} \boldsymbol{e}_{i, s}\right\},
$$

where $\varphi^{\text {post }}$ and $\Upsilon^{\text {post }}$ are given by Corollary 1.

The linearity of conditional expectations and Theorem 3 provide the ultimate claim prediction for aggregated accident years.

To quantify the prediction uncertainty we consider the conditional MSEP, e.g. defined in Wüthrich and Merz [19, Chapter 3]. The conditional MSEP, given $\mathcal{D}_{I}$, of the predictor $\widehat{C}_{J}$ for the total ultimate claim is defined by

$$
\operatorname{msep} \sum_{i=I-J+1}^{I} C_{i, J} \mid \mathcal{D}_{I}\left(\widehat{C}_{J}\right)=\mathbb{E}\left[\left(\widehat{C}_{J}-\sum_{i=I-J+1}^{I} C_{i, J}\right)^{2} \mid \mathcal{D}_{I}\right] \text {. }
$$

Due to the definition of $\widehat{C}_{J}$, see (4.1), we obtain

$$
\begin{aligned}
& \operatorname{msep} \sum_{i=I-J+1}^{I} C_{i, J} \mid \mathcal{D}_{I}\left(\widehat{C}_{J}\right)=\operatorname{Var}\left(\sum_{i=I-J+1}^{I} C_{i, J} \mid \mathcal{D}_{I}\right) \\
& =\sum_{I-J<i, l \leq I} \operatorname{Cov}\left(C_{i, J}, C_{l, J} \mid \mathcal{D}_{I}\right) \text {. }
\end{aligned}
$$

The conditional covariance terms in (4.4) can be calculated analytically and therefore the conditional MSEP attains a closed form provided in the following theorem.

Theorem 4 Under the assumptions of Model 1 we obtain for the conditional MSEP

$$
\begin{aligned}
& \operatorname{msep}_{\sum_{i=I-J+1}^{I} C_{i, J} \mid \mathcal{D}_{I}}\left(\widehat{C}_{J}\right) \\
& =\sum_{I-J<i, l \leq I} \widehat{C}_{i, J} \widehat{C}_{l, J} \sum_{s=1}^{2^{J-(I-i)}} \sum_{u=1}^{2^{J-(I-l)}} \omega_{i, s}^{\text {post }} \omega_{l, u}^{\text {post }}\left(\exp \left\{\boldsymbol{e}_{i, s}^{\prime} \Upsilon^{\text {post }} \boldsymbol{e}_{l, u}\right\}-1\right),
\end{aligned}
$$

where $\widehat{C}_{m, J}$ is given by Theorem 3 and 


$$
\begin{aligned}
\omega_{m, n}^{\text {post }} & =\frac{\beta_{m, n}^{\text {post }}}{\sum_{v=1}^{2^{J-(I-m)}} \beta_{m, v}^{\text {post }}} \in(0,1), \quad \text { and } \\
\beta_{m, n}^{\text {post }} & =\exp \left\{\boldsymbol{e}_{m, n}^{\prime} \boldsymbol{\varphi}^{\text {post }}+\frac{1}{2} \boldsymbol{e}_{m, n}^{\prime} \Upsilon^{\text {post }} \boldsymbol{e}_{m, n}\right\},
\end{aligned}
$$

for $n \in\left\{1, \ldots, 2^{J-(I-m)}\right\}$ and $m \in\{I-J, \ldots, I\}$.

Remark. Theorem 4 provides the interpretation that the conditional MSEP can be obtained as a volume weighted average of the covariance factors $\exp \left\{\boldsymbol{e}_{i, s}^{\prime} \Upsilon^{\text {post }} \boldsymbol{e}_{l, u}\right\}-1$ for log-normal distributions.

Theorem 4 allows one to derive simple upper bounds for the coefficient of variation of the ultimate claim defined by

$$
\operatorname{Vco}\left(C_{J} \mid \mathcal{D}_{I}\right)=\frac{\operatorname{Var}\left(\sum_{i=I-J+1}^{I} C_{i, J} \mid \mathcal{D}_{I}\right)^{1 / 2}}{\mathbb{E}\left[\sum_{i=I-J+1}^{I} C_{i, J} \mid \mathcal{D}_{I}\right]}=\frac{\left[\operatorname{msep}_{\sum_{i=I-J+1}^{I} C_{i, J} \mid \mathbb{D}_{I}}\left(\widehat{C}_{J}\right)\right]^{1 / 2}}{\widehat{C}_{J}} .
$$

We set $v_{i, l}^{\max }=\max _{s, u} \exp \left\{\boldsymbol{e}_{i, s}^{\prime} \Upsilon^{\text {post }} \boldsymbol{e}_{l, u}\right\}$ which is strictly greater than 1 , since $\Upsilon^{\text {post }}$ is positive definite and $v^{\max }=\max _{i, l} v_{i, l}^{\max }$. The following corollary provides a simple upper bound for $\operatorname{Vco}\left(C_{J} \mid \mathcal{D}_{I}\right)$.

Corollary 2 Under the assumptions of Model 1 we have

$$
\operatorname{Vco}\left(C_{J} \mid \mathcal{D}_{I}\right) \leq \frac{\left[\sum_{I-J<i, l \leq I} \widehat{C}_{i, J} \widehat{C}_{l, J}\left(v_{i, l}^{\max }-1\right)\right]^{1 / 2}}{\widehat{C}_{J}} \leq\left(v^{\max }-1\right)^{1 / 2}
$$

\section{Case study}

We revisit the data set for cumulative claims payments studied in Wüthrich et al. [18]. The data is provided in Table 5.1 of Wüthrich et al. [18] and fulfills the necessary condition of Model 1 that $C_{i, j+1}-C_{i, j} \geq 0$. In this case study we choose the prior covariance matrix in Model 1 to be $T=\operatorname{diag}\left(t_{0}, \ldots, t_{J}\right)$. Then, our Model 1 for $\rho=0$ (benchmark case) corresponds to the Bayes CL model studied in Wüthrich et al. [18]. Therefore, we choose the parameters $\phi_{j}, t_{j}$ and $\sigma_{j}$ for $j=1, \ldots, J$ in Model 1 to be equal to the ones given in Table 5.1 of Wüthrich et al. [18]. In addition to Wüthrich et al. [18], we also need to specify the priors for $j=0$, here. We choose for these factors vague prior parameters $\phi_{0}=9.5$ and $t_{0}=1000$ and $\sigma_{0}=0.1$ which corresponds to the rounded value of the sample standard deviation of the $\xi_{i, 0}$.

We define the total claims reserves by the conditional expectation of outstanding loss liabilities, i.e.

$$
\widehat{R}=\widehat{R}(\rho)=\mathbb{E}\left[\sum_{i=I-J+1}^{I}\left(C_{i, J}-C_{i, I-i}\right) \mid \mathcal{D}_{I}\right]=\sum_{i=I-J+1}^{I}\left(\widehat{C}_{i, J}-C_{i, I-i}\right) .
$$


Note that payments $C_{i, I-i}$ are observable at time $I$, i.e. $\mathcal{D}_{I}$-measurable. For the reserves in Model 1 in the case where accident years $i$ are assumed to behave conditionally independent, i.e. $\rho=0$, we obtain $\widehat{R}(\rho=0)=24^{\prime} 672$ (benchmark). These reserves correspond to the reserves calculated in Table 5.2 of Wüthrich et al. [18]. Moreover, they are close to the classical CL reserves $\widehat{R}^{C L}=24^{\prime} 135$ calculated according to Mack [14].

The main purpose of this paper is to study the sensitivities of the claims reserves and the corresponding prediction uncertainty as a function of the accounting year correlation parameter $\rho$ in Model 1 . Table 1 and Fig. 2 show the reserves $\widehat{R}(\rho)$ in Model 1 for increasing levels of $\rho \in[0,1)$. We observe that for this data set the reserves are almost constant in $\rho$ which says that the prediction (first moment) is only marginally affected by the correlation assumption (through Bayesian parameter estimation). Furthermore, Table 1 provides the square root of the conditional MSEP of the outstanding loss liabilities given by Theorem 4 and denoted by msep ${ }^{1 / 2}$. For large claims development triangles (i.e. $J$ large) it is simpler to directly evaluate Corollary 2.2 with MC simulation. This has also the advantage that we obtain the full predictive distribution of the outstanding loss liabilities, see Fig. 3 below.

To study the effect of accounting year correlation on the prediction uncertainty we consider msep ${ }^{1 / 2}$ of Model 1 for $\rho=0$ as the benchmark case in comparison with choices $\rho>0$. We observe a significant increase in the prediction uncertainty for increasing $\rho$. For instance, we observe an increase of more than $15 \%$ of the msep $^{1 / 2}$ by increasing $\rho$ from 0 to 0.10 and an increase of more than $31 \%$ by increasing $\rho$ from 0 to 0.20 . In terms of the relative uncertainty we observe an increase from 15.8 to $20.9 \%$ for increasing $\rho$ from 0 to 0.20 . The main reason for this is the fact that positive accounting year correlation reduces diversification benefits between accident years. We observe that while the effect on the reserves is

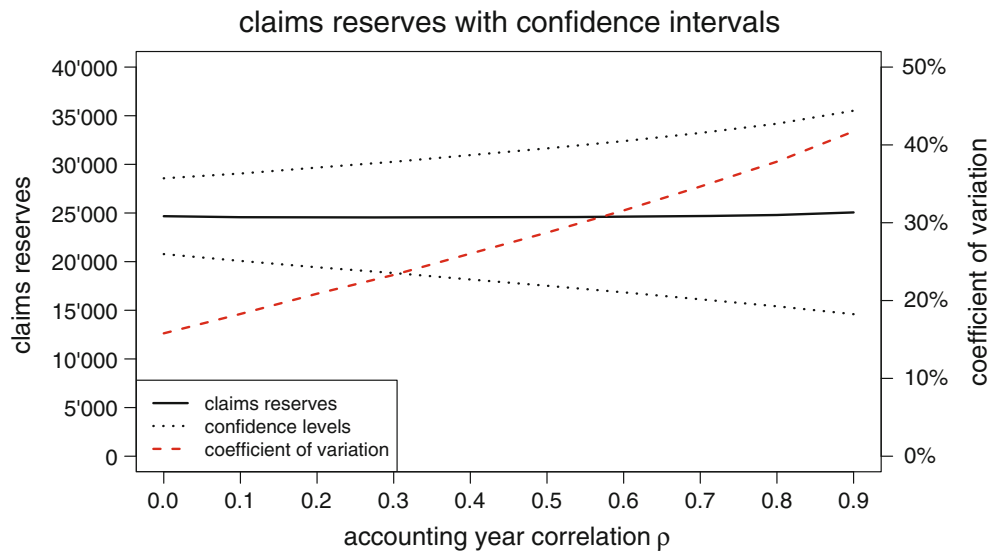

Fig. 2 Claims reserves with confidence intervals of one standard deviation measured by the square root of the conditional MSEP, msep $^{1 / 2}$, see Table 1 , and coefficient of variation (scale on the right axis) as a function of the accounting year correlation parameter $\rho \in[0,1)$ 
moderate accounting year correlation significantly contributes to the prediction uncertainty, even at low levels of positive accounting year correlation. Consequently, this implies that models assuming independence between different accident years typically underestimate the prediction uncertainty.

In practice, one often evaluates models assuming that accident years behave independently in a first step and performs ad-hoc adjustment to account for dependencies between different accident years afterwards. In particular, we rewrite the conditional MSEP of the total ultimate claim predictor $\widehat{C}_{J}$ as follows

$$
\begin{aligned}
& \operatorname{msep}_{\sum_{i=I-J+1}^{I} C_{i, J} \mid \mathcal{D}_{I}}\left(\widehat{C}_{J}\right)=\sum_{i=I-J+1}^{I} \operatorname{msep}_{C_{i, J} \mid \mathcal{D}_{I}}\left(\widehat{C}_{i, J}\right) \\
& +\sum_{I-J+1 \leq i \neq l \leq I} \operatorname{msep}_{C_{i, J} \mathcal{D}_{I}}\left(\widehat{C}_{i, J}\right)^{1 / 2} \operatorname{msep}_{C_{l, J} \mid \mathcal{D}_{I}}\left(\widehat{C}_{l, J}\right)^{1 / 2} \operatorname{Corr}\left(C_{i, J} C_{l, J} \mathcal{D}_{I}\right) \\
& =\sum_{i=I-J+1}^{I} \operatorname{msep}_{C_{i, J} \mid \mathcal{D}_{I}}\left(\widehat{C}_{i, J}\right)+\psi(\rho) \sum_{I-J+1 \leq i \neq l \leq I} \operatorname{msep}_{C_{i, J} \mid \mathcal{D}_{I}}\left(\widehat{C}_{i, J}\right)^{1 / 2} \\
& \operatorname{msep}_{C_{l, J} \mid \mathcal{D}_{I}}\left(\widehat{C}_{l, J}\right)^{1 / 2}
\end{aligned}
$$

where in the last step we substitute all the different correlation terms by the "average" implied correlation factor $\psi(\rho)$. Then we calculate and plug in all conditional MSEP of the ultimate claim for single accident years $i$ w.r.t. Model 1 for $\rho=0$. These are considered fixed for all subsequent calculations. Then by plugging in the different values for the conditional MSEP of the aggregated ultimate claim from Table 1, i.e. for different levels of $\rho$ and solving the equations for $\psi(\rho)$, we can calculate the average implied correlation factors listed in Table 2.

Note that the average implied correlation factor $\psi(\rho=0)$ is greater than 0 which is due to the fact that despite there is no correlation along accounting years for given parameters we have correlation through joint parameter uncertainty.

Table 1 Total claims reserves and the $\operatorname{msep}^{1 / 2}$ for different levels of $\rho \in[0,0.90]$

\begin{tabular}{llll}
\hline$\rho$ & Claims reserves & msep $^{1 / 2}$ & $\begin{array}{l}\text { msep } \\
\text { reserves }(\%)\end{array}$ \\
\hline 0.00 & $24^{\prime} 672$ & $3^{\prime} 888$ & 15.8 \\
0.10 & $24^{\prime} 567$ & $4^{\prime} 490$ & 18.3 \\
0.20 & $24^{\prime} 546$ & $5^{\prime} 125$ & 20.9 \\
0.30 & $24^{\prime} 547$ & $5^{\prime} 718$ & 23.3 \\
0.40 & $24^{\prime} 560$ & $6^{\prime} 393$ & 26.0 \\
0.50 & $24^{\prime} 582$ & $7^{\prime} 062$ & 28.7 \\
0.60 & $24^{\prime} 617$ & $7^{\prime} 773$ & 31.6 \\
0.70 & $24^{\prime} 678$ & $8^{\prime} 551$ & 34.7 \\
0.80 & $24^{\prime} 794$ & $9^{\prime} 384$ & 37.9 \\
0.90 & $25^{\prime} 058$ & $10^{\prime} 458$ & 41.7 \\
\hline
\end{tabular}


Table 2 Average implied correlation factors for different levels of $\rho \in[0,0.90]$

\begin{tabular}{lllllllllll}
\hline$\rho$ & 0.00 & 0.10 & 0.20 & 0.30 & 0.40 & 0.50 & 0.60 & 0.70 & 0.80 & 0.90 \\
\hline$\psi(\rho)$ & 0.004 & 0.039 & 0.081 & 0.125 & 0.182 & 0.244 & 0.316 & 0.404 & 0.506 & 0.653 \\
\hline
\end{tabular}

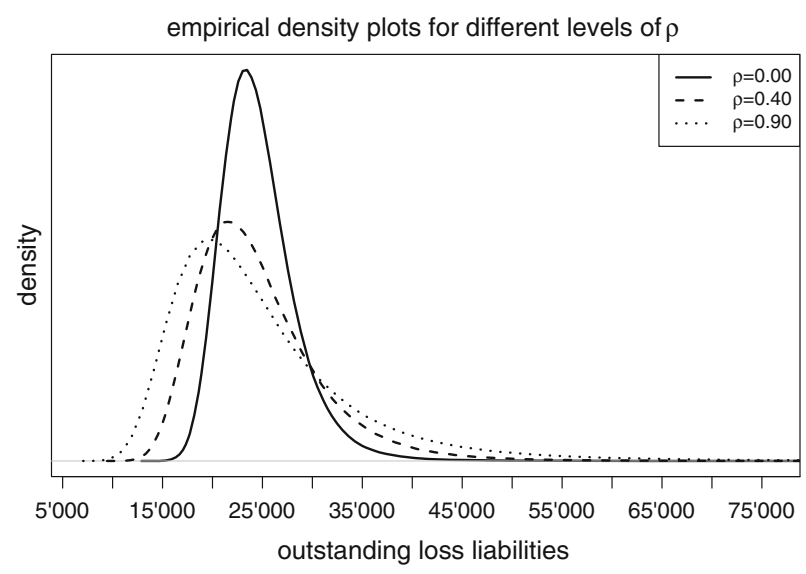

Fig. 3 Empirical densities for the outstanding loss liabilities for different levels of $\rho$

To measure the prediction uncertainty by other risk measures than the conditional MSEP we can apply MC simulations to get the full predictive distributions of the outstanding loss liabilities for Model 1. Figure 3 illustrates empirical densities for $\rho=0.0,0.4$ and 0.9. We observe that densities become more widespread for increasing $\rho$. In addition, we see that the distributions are skewed to the right and become more skewed for increasing $\rho$. In summary, the distribution of the outstanding loss liabilities becomes much more dangerous for increasing $\rho$. This implies that accounting year effects modeling substantially contributes to the prediction uncertainty leading to higher capital requirements for general insurance companies in a solvency perspective.

\section{Limitations and possible extensions}

Bayes models rely on distributional model assumptions and any further model analysis depends on this initial choice. For the multivariate Bayes CL model provided in this paper we can derive a simple parametric form for the posterior distribution of $\boldsymbol{\xi}_{\mathcal{D}_{I}^{c}}$, given $\mathcal{D}_{I}$. This allows one to derive relevant quantities, e.g. the total claims reserves and the corresponding conditional MSEP in closed form which allows for a study of parameter sensitivities. Moreover, by simple MC 
simulation we get the full predictive distribution of the outstanding loss liabilities which allows one to analyze any other risk measure. However, in practice it might be desirable to study models that go beyond joint log-Gaussianity for the ratios $C_{i, j} / C_{i, j-1}$. Bayes models with different distributional assumptions or more complex Bayes models can only be evaluated by MCMC simulation techniques, as long as we have explicit expressions for the densities. To study more general models one could further consider hierarchical generalized linear models, see e.g. Lee et al. [12] and Gigante et al. [4] (see also Remarks 1) or approaches from classical linear credibility theory, see Bühlmann and Gisler [1].

The correlation parameter $\rho$ was not calibrated from data. Our aim was to study the sensitivities of the claims reserving analysis in this correlation parameter. Calibration of $\rho$ however is a difficult task. A pure data analysis does not always lead to reasonable parameters therefore, in practice, $\rho$ is either specified by experts or additional model structure with additional economic indicators is used, see for instance Shi et al. [15] and Donnelly and Wüthrich [3].

We have assumed the same constant correlation parameter $\rho$ for all accounting years. This assumption can easily be relaxed and the main results still hold true, because the specific choice of the correlation structure is irrelevant in the proof of Theorem 2.

\section{Conclusion}

We introduce a multivariate Bayes CL ladder model that allows for the modeling of dependence along accounting years. The main purpose of the paper is to study sensitivities of the claims reserves and the corresponding prediction uncertainty as a function of the accounting year correlation parameter. This allows one to study the impact of the stringent model assumption that accident years behave independently which is common to most reserving methods. The case study shows that the prediction uncertainty significantly increases for increasing levels of correlation within accounting years. In fact, modeling positive correlation along accounting years in runoff triangles reduces diversification benefits between accident years substantially. Consequently, this implies that models assuming independence between different accident years typically underestimate the prediction uncertainty.

\section{A. Appendix}

Proof of Theorem 2 For the proof of Theorem 2 we consider the moment generating function of $\boldsymbol{\xi}$. To do so, we choose $\boldsymbol{a}=\left(\boldsymbol{a}^{(1)^{\prime}}, \ldots, \boldsymbol{a}^{(I+J)^{\prime}}\right)^{\prime} \in \mathbb{R}^{I(J+1)}$ with $\boldsymbol{a}^{(k)} \in \mathbb{R}^{q}$ for all $k=1, \ldots, I+J$. Then we obtain for the moment generating function of $\boldsymbol{\xi}$ (using the conditional independence of the $\xi^{(k)}$ in the fourth equation) 


$$
\begin{aligned}
M_{\boldsymbol{\xi}}(\boldsymbol{a}) & =\mathbb{E}\left[\exp \left\{\boldsymbol{a}^{\prime} \boldsymbol{\xi}\right\}\right]=\mathbb{E}\left[\mathbb{E}\left[\exp \left\{\sum_{k=1}^{I+J} \boldsymbol{a}^{(k)^{\prime}} \boldsymbol{\xi}^{(k)}\right\} \mid \boldsymbol{\mu}\right]\right] \\
& =\mathbb{E}\left[\mathbb{E}\left[\prod_{k=1}^{I+J} \exp \left\{\boldsymbol{a}^{(k)^{\prime}} \boldsymbol{\xi}^{(k)}\right\} \mid \boldsymbol{\mu}\right]=\mathbb{E}\left[\prod _ { k = 1 } ^ { I + J } \operatorname { e x p } \left\{\boldsymbol{a}^{(k)^{\prime}} P_{k} \boldsymbol{\mu}+\frac{1}{2} \boldsymbol{a}^{\left.\left.(k)^{\prime} \Sigma_{k} \boldsymbol{a}^{(k)}\right\}\right]}\right.\right.\right. \\
& =\mathbb{E}\left[\exp \left\{\left(\sum_{k=1}^{I+J} \boldsymbol{a}^{(k)^{\prime}} P_{k}\right) \boldsymbol{\mu}\right\}\right] \exp \left\{\frac{1}{2} \sum_{k=1}^{I+J} \boldsymbol{a}^{\left.(k)^{\prime} \sum_{k} \boldsymbol{a}^{(k)}\right\}}\right\} \\
& =\exp \left\{\sum_{k=1}^{I+J} \boldsymbol{a}^{(k)^{\prime}} \boldsymbol{P}_{k} \boldsymbol{\phi}+\frac{1}{2}\left(\sum_{k=1}^{I+J} \boldsymbol{a}^{(k)^{\prime}} P_{k}\right) T\left(\sum_{l=1}^{I+J} \boldsymbol{a}^{(l){ }^{\prime}} P_{l}\right)^{\prime}+\frac{1}{2} \sum_{k=1}^{I+J} \boldsymbol{a}^{(k)^{\prime}} \Sigma_{k} \boldsymbol{a}^{(k)}\right\} \\
& =\exp \left\{\sum_{k=1}^{I+J} \boldsymbol{a}^{(k)^{\prime}}\left(P_{k} \boldsymbol{\phi}\right)+\frac{1}{2} \sum_{k, l=1}^{I+J} \boldsymbol{a}^{(k)^{\prime}}\left(P_{k} T P_{l}^{\prime}+\sum_{k} 1_{\{k=l\}}\right) \boldsymbol{a}^{(l)}\right\} \\
& =\exp \left\{\boldsymbol{a}^{\prime} \boldsymbol{\varphi}+\frac{1}{2} \boldsymbol{a}^{\prime} \Upsilon \boldsymbol{a}\right\},
\end{aligned}
$$

where $\varphi$ and $\Upsilon$ are defined in Theorem 2. Therefore, the unconditional distribution of $\xi$ is multivariate normal with parameters $\varphi$ and $\Upsilon$. This completes the proof.

Proof of Theorem 3 Formula (4.3) and Corollary 1 imply that

$$
\begin{aligned}
\widehat{C}_{i, J} & =\mathbb{E}\left[C_{i, J} \mid \boldsymbol{\xi}_{\mathcal{D}_{I}}\right]=C_{i, I-i} \sum_{s=1}^{2^{J-(I-i)}} \mathbb{E}\left[\exp \left\{\boldsymbol{e}_{i, s}^{\prime} \boldsymbol{\xi}_{\mathcal{D}_{I}^{c}}\right\} \mid \boldsymbol{\xi}_{\mathcal{D}_{I}}\right] \\
& =C_{i, I-i} \sum_{s=1}^{2^{J-(I-i)}} \exp \left\{\boldsymbol{e}_{i, s}^{\prime} \boldsymbol{\varphi}^{\text {post }}+\frac{1}{2} \boldsymbol{e}_{i, s}^{\prime} \Upsilon^{\text {post }} \boldsymbol{e}_{i, s}\right\},
\end{aligned}
$$

where the last equation is a direct consequence of log-normal distributions.

Proof of Theorem 4 By applying Corollary 1 and using the notation introduced in Theorem 3 we obtain for the covariance terms in formula (4.4)

$$
\begin{aligned}
\operatorname{Cov}\left(C_{i, J}, C_{l, J} \mid \mathcal{D}_{I}\right) & =C_{i, I-i} C_{l, I-l} \sum_{s=1}^{2^{J-(I-i)}} \sum_{u=1}^{2^{J-(I-l)}} \operatorname{Cov}\left(\exp \left\{\boldsymbol{e}_{i, s}^{\prime} \boldsymbol{\xi}_{\mathcal{D}_{I}^{c}}\right\}, \exp \left\{\boldsymbol{e}_{l, u}^{\prime} \boldsymbol{\xi}_{\mathcal{D}_{I}^{c}}\right\} \boldsymbol{\xi}_{\mathcal{D}_{I}}\right) \\
& =C_{i, I-i} C_{l, I-l} \sum_{s=1}^{2^{J-(I-i)}} \sum_{u=1}^{2^{J-(I-l)}} \exp \left\{\boldsymbol{e}_{i, s}^{\prime} \boldsymbol{\varphi}^{\text {post }}+\frac{1}{2} \boldsymbol{e}_{i, s}^{\prime} \Upsilon^{\text {post }} \boldsymbol{e}_{i, s}\right\} \\
& \times \exp \left\{\boldsymbol{e}_{l, u}^{\prime} \boldsymbol{\varphi}^{\text {post }}+\frac{1}{2} \boldsymbol{e}_{l, u}^{\prime} \Upsilon^{\text {post }} \boldsymbol{e}_{l, u}\right\}\left(\exp \left\{\boldsymbol{e}_{i, s}^{\prime} \Upsilon^{\text {post }} \boldsymbol{e}_{l, u}\right\}-1\right) .
\end{aligned}
$$

By substituting $\beta_{m, n}^{\text {post }}=\exp \left\{\boldsymbol{e}_{m, n}^{\prime} \boldsymbol{\varphi}^{\text {post }}+\frac{1}{2} \boldsymbol{e}_{m, n}^{\prime} \Upsilon^{\text {post }} \boldsymbol{e}_{m, n}\right\}$ we obtain for the conditional MSEP 


$$
\begin{aligned}
& \sum_{I-J<i, l \leq I} C_{i, I-i} C_{l, I-l} \sum_{s=1}^{2^{J-(I-i)}} \sum_{u=1}^{J-(I-l)} \beta_{i, s}^{\text {post }} \beta_{l, u}^{\text {post }}\left(\exp \left\{\boldsymbol{e}_{i, s}^{\prime} \Upsilon^{\text {post }} \boldsymbol{e}_{l, u}\right\}-1\right) \\
& =\sum_{I-J<i, l \leq I} \widehat{C}_{i, J} \widehat{C}_{l, J} \sum_{s=1}^{2^{J-(I-i)}} \sum_{u=1}^{2^{J-(I-l)}} \frac{\beta_{i, s}^{\text {post }}}{\sum_{v=1}^{2^{J-(I-i)}} \beta_{i, v}^{\text {post }} \sum_{w=1}^{2^{J-(I-l)}} \beta_{l, w}^{\text {post }}}\left(\exp \left\{\boldsymbol{e}_{i, s}^{\prime} \Upsilon^{\text {post }} \boldsymbol{e}_{l, u}\right\}-1\right) .
\end{aligned}
$$

In the last equation we multiply and divide each term $C_{m, I-m}$ by $\sum_{n=1}^{2^{J-(I-m)}} \beta_{m, n}^{\text {post }}$ and set, see Theorem 3,

$$
\widehat{C}_{m, J}=C_{m, I-m} \sum_{n=1}^{2^{J-(I-m)}} \beta_{m, n}^{\text {post }} .
$$

Substituting $\omega_{m, n}^{\text {post }}$ as defined in Theorem 4 provides the claim.

Proof of Corollary 2 For the coefficient of variation of the ultimate claim we obtain

$$
\begin{aligned}
\operatorname{Vco}\left(C_{J} \mid \mathcal{D}_{I}\right) & \\
= & \frac{\left[\sum_{I-J<i, l \leq I} \widehat{C}_{i, J} \widehat{C}_{l, J} \sum_{s=1}^{2^{J-(I-i)}} \sum_{u=1}^{2^{J-(I-l)}} \omega_{i, s}^{\text {post }} \omega_{l, u}^{\text {post }}\left(\exp \left\{\boldsymbol{e}_{i, s}^{\prime} \Upsilon^{\text {post }} \boldsymbol{e}_{l, u}\right\}-1\right)\right]^{1 / 2}}{\sum_{I-J<i \leq I} \widehat{C}_{i, J}} \\
& \leq \frac{\left[\sum_{I-J<i, l \leq I} \widehat{C}_{i, J} \widehat{C}_{l, J} \sum_{s=1}^{2^{J-(I-i)}} \sum_{u=1}^{2^{J-(I-l)}} \omega_{i, s}^{\text {post }} \omega_{l, u}^{\text {post }}\left(v_{i, l}^{\max }-1\right)\right]^{1 / 2}}{\sum_{I-J<i \leq I} \widehat{C}_{i, J}} \\
& =\frac{\left[\sum_{I-J<i, l \leq I} \widehat{C}_{i, J} \widehat{C}_{l, J}\left(v_{i, l}^{\max }-1\right)\right]^{1 / 2}}{\widehat{C}_{J}} .
\end{aligned}
$$

The last step follows because $\sum_{n=1}^{2^{J-(l-m)}} \omega_{m, n}^{\text {post }}=1$. The second upper bound for $\operatorname{Vco}\left(C_{J} \mid \mathcal{D}_{I}\right)$ follows directly from

$$
\operatorname{Vco}\left(C_{J} \mid \mathcal{D}_{I}\right) \leq \frac{\left[\sum_{I-J<i, l \leq I} \widehat{C}_{i, J} \widehat{C}_{l, J}\left(v_{i, l}^{\max }-1\right)\right]^{1 / 2}}{\widehat{C}_{J}} \leq\left(v^{\max }-1\right)^{1 / 2}
$$

\section{References}

1. Bühlmann H, Gisler A (2005) A course in credibility theory and its applications. Springer, Berlin

2. Clark DR (2006) Variance and covariance due to inflation. CAS Forum (fall), pp 61-95

3. Donnelly C, Wüthrich MV (2012) Bayesian prediction of disability insurance frequencies using economic factors. Ann Actuar Sci (to appear)

4. Gigante P, Picech L, Sigalotti L (2012) Claims reserving in the hierarchical generalised linear models framework (preprint) 
5. Hertig J (1985) A statistical approach to the IBNR-reserves in marine insurance. Astin Bull 15(2):171-183

6. Jessen AH, Rietdorf N (2011) Diagonal effects in claims reserving. Scand Actuar J 1:21-37

7. de Jong P (2006) Forecasting runoff triangles. North Am Actuar J 10(2):28-38

8. Johnson RA, Wichern DW (1988) Applied multivariate statistical analysis, 2nd edn. Prentice Hall, Upper Saddle River

9. Kuang D, Nielsen B, Nielsen JP (2008) Identification of the age-period-cohort model and the extended chain-ladder model. Biometrika 95:979-986

10. Kuang D, Nielsen B, Nielsen JP (2008) Forecasting with the age-period-cohort model and the extended chain-ladder model. Biometrika 95:987-991

11. Kuang D, Nielsen B, Nielsen JP (2011) Forecasting in an extended chain-ladder-type model. J Risk Insur 78(2):345-359

12. Lee Y, Nelder JA, Pawitan P (2006) Unified analysis via H-likelihood. Generalized linear models with random effects. Chapman\& Hall, Boca Raton

13. McNeil AJ, Frey R, Embrechts P (2005) Quantitative risk management: concepts, techniques. Princeton University Press, Princeton

14. Mack T (1993) Distribution-free calculation of the standard error of chain ladder reserve estimates. Astin Bull 23(2):213-225

15. Shi P, Basu S, Meyers GG (2012) A Bayesian log-normal model for multivariate loss reserving. North Am Actuar J 16(1):29-51

16. Venter GG (2007) Refining reserve runoff ranges. CAS Forum (summer)

17. Wüthrich MV (2010) Accounting year effects modeling in the stochastic chain ladder reserving method. North Am Actuar J 14(2):235-255

18. Wüthrich MV, Embrechts P, Tsanakas A (2011) Risk margin for a non-life insurance run-off. Stat Risk Model 28(4):299-317

19. Wüthrich MV, Merz M (2008) Stochastic claims reserving methods in insurance. Wiley, New York 